\title{
UMBRALES DE LLUVIA, DAÑOS Y NIVELES DE ALERTA EN LA COMUNIDAD VALENCIANA
}

\author{
Ana M. CAMARASA-BELMONTE¹, Damián BUTRÓN¹ \\ ${ }^{1}$ Departamento de Geografia. Universidad de Valencia \\ Avda. Blasco Ibañez, 28. 46010. Valencia (+34963864237). \\ ana.camarasa@uv.es, daburo@alumni.uv.es
}

\section{RESUMEN}

En el contexto de los riesgos climáticos, muchas de las alarmas tempranas de precipitaciones extremas se basan en umbrales de lluvia que, a su vez, están relacionados con niveles probables de daños. A pesar de los grandes esfuerzos que se han venido realizando en los últimos años para discriminar bien los umbrales de estas alertas, uno de los principales problemas es la emisión de un número excesivo de avisos, hecho que puede crear alarmismo.

El presente trabajo constituye un estudio aplicado que aborda la detección de indicadores de lluvia y la estimación de sus umbrales de riesgo en la Comunidad Valenciana, entre 1997 y 2007. Se basa en el estudio comparativo entre dos bases de datos: (1) una pluviométrica desarrollada en la Universidad de Valencia a partir de los datos del Sistema Automático de Información Hidrológica, y (2) la otra, sobre daños por inundación en la Comunidad Valenciana, elaborada por el Servicio de Protección Civil. Además evalúa la adecuación de los umbrales de alarma vigentes en el Plan Meteoalerta.

Palabras clave: alerta meteorológica, umbral de lluvia, riesgo, daños.

\begin{abstract}
Many of the early weather warning systems are based on precipitation thresholds, which, in turn, are related to levels of damage. Despite the great efforts recently made in order to discriminate these thresholds, one of the main problems is the excessive number of alert bulletins that can create alarmism. This work consists of an applied study that addresses the detection of rainfall indicators and their risk thresholds in Valencia, between 1997 and 2007. It is based on the comparison of two databases: (1) a rainfall database, developed at the University of Valencia from the Automatic Hydrological Information System, and (2) a flood damage database, provided by the Civil Defense Department. The accuracy of the current alarm thresholds in the Meteoalerta Plan is also assessed.
\end{abstract}

Key words: meteorological warming system, rainfall threshold, risk, damage.

\section{INTRODUCCIÓN}

La Comunidad Valenciana lidera el ranking de pérdidas por inundación en España según el Consorcio de Compensación de Seguros (2014). A pesar del todos los esfuerzos para frenar las pérdidas, el problema, lejos de paliarse con el tiempo, tiende a 
agudizarse. Ante este panorama poco esperanzador, los organismos públicos se afanan por diseñar estrategias que permitan prevenir el riesgo con suficiente antelación. En España, una de las acciones más significativa que se ha venido desarrollando en las últimas décadas ha sido el Plan Nacional de Predicción y Vigilancia de Fenómenos Meteorológicos Adversos: Meteoalerta (PNPVFA), operativo desde 2006. Se trata de un plan llevado a cabo desde la Agencia Estatal de Meteorología (AEMET), que tiene su origen en los primitivos planes PREVIMET (años 80) y que actualmente converge con el proyecto europeo Meteoalarm (Elizaga, 2009). Su principal objetivo es facilitar a todos los ciudadanos e instituciones públicas, la mejor y más actualizada información sobre fenómenos atmosféricos adversos, con una antelación de hasta sesenta horas, así como un seguimiento de su evolución.

Para ello, una de las primeras medidas en que se basa el Plan es la emisión de boletines de aviso, cuyo nivel de peligrosidad se establece a partir de umbrales de intensidad y duración de cada fenómeno (AEMET, 2009). Por lo general se establecen cuatro categorías, caracterizadas por colores, que van desde el verde (sin riesgo) hasta el rojo (riesgo extremo), pasando por el amarillo (riesgo bajo) y el naranja (riesgo importante).

A pesar de los grandes esfuerzos que se ha venido realizando en los últimos años para discriminar bien los umbrales de estas alertas, uno de los principales problemas, que se arrastra ya desde planes anteriores, es la emisión de un número excesivo de avisos (Elizaga, 2009). La tarea resulta muy difícil, máxime cuando los boletines atañen a fenómenos de lluvia intensa y en el ámbito de la vertiente mediterránea (Lorente et al., 2013). Después de varias décadas de desarrollo de los sistemas de alerta temprana, el acierto de los pronósticos de fenómenos adversos de lluvia es prácticamente del $100 \%$. Sin embargo, en la mayoría de los casos no se producen daños relevantes. No se trata, por tanto, de un problema de acierto de los pronósticos meteorológicos, sino de un ajuste deficiente de los umbrales de alarma.

Pese a todas estas incertidumbres, los planes de previsión no pueden dejar de contar con unos umbrales que permitan, de una manera sencilla y ágil, establecer categorías con las que abordar los fenómenos adversos, en relación al riesgo.

El presente trabajo analiza la adecuación de los umbrales de alarma del Plan Meteoalerta al riesgo realmente acaecido en la Comunidad Valenciana, entre 1997 y 2007, con ocasión de episodios de lluvia extrema. Para ello se basa en el estudio comparativo entre dos bases de datos: (1) una de tipo pluviométrico desarrollada en la Universidad de Valencia (proyecto RAINMED) a partir de los datos del SAIH-Júcar y (2) la otra, sobre daños por inundación en la Comunidad Valenciana, elaborada por el Servicio de Protección Civil. Constituye un estudio aplicado que permite seleccionar qué parámetros pluviométricos pueden ser considerados como indicadores de riesgo y con qué umbrales se producen pérdidas.

\section{2. ÁREA DE ESTUDIO Y DATOS}

El área de estudio abarca la práctica totalidad de la Comunidad Valenciana. Sin embargo, el hecho de que los datos de partida provengan de dos bases de datos, organizadas espacialmente de manera diferente, obliga a una explicación más detallada. Como ya se ha comentado en el apartado anterior, los daños provienen de Protección 
Civil y están desagregados a nivel municipal, mientras que la información de pluviometría corresponde a la base de datos sobre episodios de lluvia generados en el seno del proyecto RAINMED, desarrollado en la Universidad de Valencia. Este proyecto abarca el territorio de la Confederación Hidrográfica del Júcar (CHJ), dividido en 11 unidades espaciales homogéneas (denominadas zonas), siguiendo criterios topográficos e hidrológicos (Camarasa y López, 2006). Siete de las once unidades afectan a la Comunidad Valenciana (Castellón, Valencia, Benageber, Alzira, Cofrentes, Alicante y Gandía) y en este trabajo han sido utilizadas como referencia espacial de ubicación de los episodios de lluvia (figura 1).

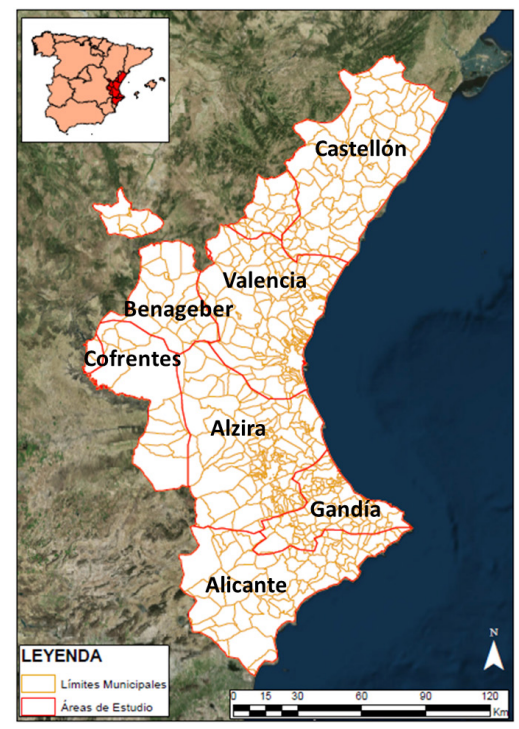

Fig. 1. Área de estudio (límites municipales y áreas pluviométricas)

\section{MÉTODO}

La metodología se basa en relacionar los daños registrados en la Comunidad Valenciana por episodios extremos con las características de las lluvias que los han producido. Asímismo, con objeto de evaluar el grado de adecuación de los umbrales de riesgo del Plan Meteoalerta, se compararán los daños realmente registrados por Protección Civil con los esperables según las previsiones de dicho Plan.

La primera dificultad que presenta este análisis deriva de la diferente naturaleza de la información de partida: cuantitativa, en todo lo referente a los indicadores pluviométricos, y básicamente cualitativa, en lo referente a los daños. Para relacionar ambas bases de datos, la metodología aplicada en este trabajo ha seguido las siguientes fases: en primer lugar se han clasificado los daños por categorías y se han valorado en términos cuantitativos. En segundo lugar, se han puesto en relación ambas bases de datos, con objeto de seleccionar qué parámetros pluviométricos pueden considerarse indicadores de riesgo (y por encima de qué umbrales) y cuales deben de despreciarse, por no presentar correlación con los daños. Por último se ha analizado la adecuación 
de los umbrales de alerta, comparando las veces que, habiéndose alcanzado los diferentes umbrales de riesgo para 1 y 12 horas del Plan Meteoalerta, se han producido daños con las veces que no se han producido. La última fase incluye la propuesta de umbrales de alerta más ajustados a la realidad.

\subsection{Valoración de los daños entre 1997 y 2007}

La información sobre daños por municipio elaboradas por Protección Civil requiere de la categorización y valoración previa de los daños, en términos cuantitativos. Para ello se han seguido tres etapas: a) clasificación de los daños en diferentes categorías; b) ponderación de las categorías de daños; y c) valoración de los daños (tanto por categoría, como los totales del episodio).

a) Clasificación de los daños por categoría

La clasificación de los daños en diferentes categorías se inspira en el PATRICOVA (Plan de Acción Territorial de Prevención de Riesgos de Inundación en la Comunidad Valenciana), y distingue entre:

1. Personales, sobre la vida humana (evacuados, heridos, fallecidos, etc).

2. Núcleos urbanos (fundamentalmente de tipo económico).

3. Infraestructuras, básicamente de transporte y comunicación (de tipo funcional).

4. Riesgos Asociados (incendios, viento y granizo).

b) Ponderación de las categorías

Una vez establecidas las categorías de los daños se procedió a la ponderación de las mismas mediante el Método de Comparación por Pares (Saaty, 1988), cuya finalidad es identificar la importancia relativa de cada categoría respecto a las demás. Los resultados obtenidos en la matriz de comparación otorgan la mayor importancia a la categoría de daños personales (con un valor de ponderación de 7), seguida de los daños en núcleos urbanos (4), para continuar en los pérdidas en infraestructuras (2) y, por último, los impactos de los riesgos asociados (1).

c) Valoración de los daños

Una vez establecido el factor de ponderación de las categorías se evaluó el nivel de daño dentro de cada una de ellas, en una escala de 1 a 10, a partir de la descripción que figura en el informe de Protección Civil. De este modo se obtuvieron estimaciones numéricas, tanto de los daños parciales (por categorías) como generales, por episodio. Estos resultados no deben interpretarse, no obstante, como valores cuantitativos en toda su dimensión, sino más bien como magnitudes de referencia, que nos permiten comparar daños de diferente naturaleza y ponerlos en relación con los indicadores pluviométricos.

Con el objeto de analizar los daños de los diferentes episodios de una manera estandarizada, las pérdidas se han re-escalado, referenciándolas al episodio que mayores pérdidas ha causado (octubre de 2003). El nivel de daños se expresa, por tanto, como un porcentaje de ese máximo alcanzado.

\subsection{Selección de indicadores pluviométricos de referencia y estimación de umbrales de riesgo}

Una vez valorados los daños en términos cuantitativos se han puesto en relación con los indicadores pluviométricos, mediante un análisis de correlación entre la mag- 
nitud de las pérdidas (por categorías y para el total de los episodios) y el valor de los diferentes indicadores, en las zonas de referencia. Este análisis ha permitido diferenciar qué parámetros pluviométricos pueden constituir un indicador de riesgo y a partir de qué umbrales mínimos se producen daños.

\subsection{Evaluación de los niveles de alarma en el Plan Meteoalerta}

El Plan Meteoalerta discrimina la peligrosidad de un episodio a partir de cuatro niveles de alarma (verde, amarillo, naranja y rojo), en función de la intensidad de lluvia en 1 y 12 horas. Los umbrales para la Comunidad Valenciana son los siguientes (AEMET, 2009):

a) Nivel verde: no existe riesgo meteorológico.

b) Nivel amarillo: fenómenos habituales pero potencialmente peligrosos. Los umbrales de alerta son $20 \mathrm{~mm}$ en 1 hora y $60 \mathrm{~mm}$ en 12 horas.

c) Nivel naranja: fenómenos no habituales con cierto grado de peligro para las actividades usuales. Los umbrales de alerta son $40 \mathrm{~mm}$ en 1 hora y 100 en 12 horas.

d) Nivel rojo: fenómenos no habituales, de intensidad excepcional y con un nivel de riesgo muy alto. Los umbrales de alerta son $90 \mathrm{~mm}$ en 1 h y $180 \mathrm{~mm}$ en 12 horas.

Con objeto de estimar la adecuación de los umbrales del Plan Meteoralerta a la realidad, se ha realizado una comparación entre las ocasiones en que, habiéndose alcanzado dichos umbrales para $1 \mathrm{~h}$ y $12 \mathrm{~h}$, se han producido daños y las que no.

\section{RESULTADOS Y DISCUSIÓN}

\subsection{Indicadores pluviométricos de riesgo}

Las correlaciones entre los indicadores pluviométricos y los tipos de daños, ponen de manifiesto que Irregularidad y Persistencia presentan un coeficiente muy bajo $\left(\mathrm{r}^{2}<0.2\right)$, por lo que han sido rechazados como estimadores de riesgo. El resto de indicadores muestran diferentes grados de correlación, como puede verse en la tabla 1. En general, existen fuertes relaciones entre los daños por episodio y los indicadores pluviométricos seleccionados. A nivel global, los mayores coeficientes aparecen ligados a la precipitación acumulada total (valor máximo); a los valores medios de las lluvias acumuladas en $6 \mathrm{~h}$ y $12 \mathrm{~h}$ y a la intensidad horaria (valor máximo). Las correlaciones más débiles corresponden a la intensidad media y máxima cincominutales.

Estos resultados ponen de manifiesto la importancia de la lluvia acumulada al final de la tormenta y para periodos horarios largos (12 horas). La intensidad cincominutal no es indicadora de daños, porque puede darse el hecho de que se produzcan intensidades muy altas, pero mantenidas durante muy poco tiempo, acumulando, por tanto, poca agua. En un estudio empírico llevado a cabo por Camarasa-Belmonte y Soriano (2014) sobre la intensidad de lluvia a diferentes escalas se pone de manifiesto cómo las mayores intensidades, asociadas a células convectivas, se alcanzan en períodos inferiores a 1 hora. Si estas células no son muy frecuentes, la cantidad de agua que acumulan al final de la tormenta no es suficiente para provocar daños generales. No obstante sí que pueden ocasionar daños puntuales importantes, como lo demuestra el hecho de que los mayores valores de correlación en el caso de intensidades horarias, se alcancen en los registros máximos $\left(\mathrm{r}^{2}=0.82\right)$, mientras que los medios pierden 
relevancia $\left(\mathrm{r}^{2}=0.71\right)$. A partir de las 6 horas de lluvia, la tendencia sufre un punto de inflexión (Camarasa et al., 2010) y, si la tormenta continúa, pueden comenzar a verse afectadas las infraestructuras. Cuando se superan las 12 horas de lluvia a intensidades medias considerables, los daños pueden extenderse a los núcleos urbanos y a las personas. A partir de las 24 horas la importancia de la intensidad decrece frente a la de la lluvia acumulada.

\begin{tabular}{|c|c|c|c|c|c|c|c|c|c|c|c|c|}
\hline \multirow[t]{2}{*}{ DAÑOS } & \multicolumn{2}{|c|}{$\begin{array}{c}\text { LLUVIA } \\
\text { ACUMULADA } \\
\text { EN EL } \\
\text { EPISODO }\end{array}$} & \multicolumn{2}{|c|}{$\begin{array}{c}\text { INTENSIDAD } \\
\text { MÁXIMA } \\
\text { CINCOMINUTAL }\end{array}$} & \multicolumn{2}{|c|}{$\begin{array}{c}\text { INTENSIDAD } \\
\text { MEDIA } \\
\text { CINCOMINUTAL }\end{array}$} & \multicolumn{2}{|c|}{$\begin{array}{l}\text { INTENS. } \\
\text { LLUVIA } \\
\text { EN } 1 \mathrm{~h}\end{array}$} & \multicolumn{2}{|c|}{$\begin{array}{l}\text { LLUVIA } \\
\text { ACUMUL. } \\
\text { EN } 6 \mathrm{~h}\end{array}$} & \multicolumn{2}{|c|}{$\begin{array}{c}\text { LLUVIA } \\
\text { ACUMUL } \\
\text { EN } 12 \mathrm{~h}\end{array}$} \\
\hline & Max. & Med. & Max. & Med. & Max. & Med. & Max. & Med. & Max & Med & Max. & Med. \\
\hline Daños globales & 0.92 & 0.80 & 0.68 & 0.69 & 0.64 & 0.61 & 0.82 & 0.71 & 0.81 & 0.86 & 0.80 & 0.84 \\
\hline Daños urbanos & 0.84 & 0.77 & 0.46 & 0.70 & 0.35 & 0.70 & 0.75 & 0.74 & 0.74 & 0.75 & 0.73 & 0.82 \\
\hline $\begin{array}{l}\text { Daños en } \\
\text { infraestructuras }\end{array}$ & 0.84 & 0.84 & 0.55 & 0.60 & 0.52 & 0.57 & 0.70 & 0.77 & 0.81 & 0.83 & 0.82 & 0.77 \\
\hline Daños personales & 0.80 & 0.81 & 0.63 & 0.52 & 0.37 & 0.70 & 0.71 & 0.71 & 0.73 & 0.75 & 0.76 & 0.84 \\
\hline
\end{tabular}

Tabla 1: Resultados de las correlaciones $\left(r^{2}\right)$ entre la magnitud de los daños y los indicadores pluviométricos. Fuente: elaboración propia a partir de los datos de daños del Servicio de Protección Civil de la Delegación del Gobierno en la Comunidad Valenciana y de datos del SAIH-Júcar.

\subsection{Umbrales de riesgo}

Tal y como se explicó en el apartado de metodología, los daños han sido normalizados en relación porcentual al suceso que mayores pérdidas presentó y clasificados en tres intervalos $(25 \%, 50 \%$ y $75 \%)$, que podrían hacerse corresponder con los niveles de alerta propuestos por la AEMET (amarillo, naranja y rojo).

Una vez referenciados los episodios según un nivel de daño estándar, se han puesto en relación con los indicadores pluviométricos seleccionados en el apartado anterior, para ver, a partir de qué umbrales mínimos se producen daños. Se han desestimado los indicadores de intensidad cincominutal (por no resultar representativos) y se han analizado los de precipitación acumulada total, en $1 \mathrm{~h}$, en $6 \mathrm{~h}$ y en $12 \mathrm{~h}$. La tabla 2 muestra los resultados. En la última fila de la tabla aparecen los umbrales máximos de referencia, estimados por Camarasa y Soriano (2014) en el área de la Confederación Hidrográfica del Júcar.

En términos globales, con lluvias acumuladas de hasta $130 \mathrm{~mm}$ en un episodio se pueden producir daños leves; hasta $298 \mathrm{~mm}$ se esperan daños moderados y, por encima de estas cifras los daños son fuertes (con precipitaciones mayores a $466 \mathrm{~mm}$ ). Si tomamos como referencia las máximas en una hora, lluvias de más de $33 \mathrm{~mm}$ pueden comenzar a presentar problemas, aunque las pérdidas moderadas o fuertes se registran a partir de los $60 \mathrm{~mm}$ y $86 \mathrm{~mm}$ respectivamente. Las cantidades acumuladas en 6 horas que producen los daños moderados están por encima de los $114 \mathrm{~mm}$ (más de $168 \mathrm{~mm}$ si hablamos de daños fuertes), aunque con $60 \mathrm{~mm}$ ya pueden producirse daños leves. En cuanto a las precipitaciones en 12 horas, máximas de $72 \mathrm{~mm}$ empiezan a producir problemas leves, por encima de $156 \mathrm{~mm}$, moderados, y graves si superan los $228 \mathrm{~mm}$. 
Si realizamos el análisis por categorías, la mayor parte de indicadores presentan los umbrales más bajos de daño para el caso de núcleos urbanos, seguido de daños personales y por último de daños en las infraestructuras. Así, mientras que con 115 mm de lluvia acumulada al final del episodio se pueden producir daños leves en los núcleos urbanos, se necesitan más de $191 \mathrm{~mm}$ para que afecten a las personas y más de $221 \mathrm{~mm}$ para que se resientan las infraestructuras.

\begin{tabular}{|c|c|c|c|c|}
\hline \multirow[t]{2}{*}{ DAÑOS } & \multicolumn{4}{|c|}{$\begin{array}{c}\text { UMBRALES DE PRECIPITACIÓN MÁXIMA } \\
\text { ACUMULADA }(\mathrm{mm})\end{array}$} \\
\hline & Total del episodio & 1 hora & 6 horas & 12 horas \\
\hline Daños Globales & $\begin{array}{c}\mathbf{R}^{2} \\
(0.92)\end{array}$ & $\begin{array}{c}\mathbf{R}^{2} \\
(0.82)\end{array}$ & $\begin{array}{c}\mathbf{R}^{2} \\
(0.81)\end{array}$ & $\begin{array}{c}\mathbf{R}^{2} \\
(0.80)\end{array}$ \\
\hline $25 \%$ & 130 & 33 & 60 & 72 \\
\hline $50 \%$ & 298 & 60 & 114 & 156 \\
\hline $75 \%$ & 466 & 86 & 168 & 228 \\
\hline $\begin{array}{c}\text { Daños en núcleos } \\
\text { urbanos }\end{array}$ & $\begin{array}{c}\mathbf{R}^{2} \\
(0.84)\end{array}$ & $\begin{array}{c}\mathbf{R}^{2} \\
(0.75) \\
\end{array}$ & $\begin{array}{c}\mathbf{R}^{2} \\
(0.74) \\
\end{array}$ & $\begin{array}{c}\mathbf{R}^{2} \\
(0.73) \\
\end{array}$ \\
\hline $25 \%$ & 115 & 30 & 66 & 72 \\
\hline $50 \%$ & 274 & 55 & 144 & 168 \\
\hline $75 \%$ & 433 & 81 & 222 & 264 \\
\hline $\begin{array}{c}\text { Daños en } \\
\text { infraestructuras }\end{array}$ & $\begin{array}{c}\mathbf{R}^{2} \\
(0.84)\end{array}$ & $\begin{array}{c}\mathbf{R}^{2} \\
(0.70)\end{array}$ & $\begin{array}{c}\mathbf{R}^{2} \\
(0.81)\end{array}$ & $\begin{array}{c}\mathbf{R}^{2} \\
(0.82)\end{array}$ \\
\hline $25 \%$ & 221 & 43 & 90 & 96 \\
\hline $50 \%$ & 413 & 69 & 162 & 168 \\
\hline $75 \%$ & 606 & 96 & 234 & 228 \\
\hline $\begin{array}{c}\text { Daños } \\
\text { personales }\end{array}$ & $\begin{array}{c}\mathbf{R}^{2} \\
(0.80)\end{array}$ & $\begin{array}{c}\mathbf{R}^{2} \\
(0.71)\end{array}$ & $\begin{array}{c}\mathbf{R}^{2} \\
(0.73) \\
\end{array}$ & $\begin{array}{c}\mathbf{R}^{2} \\
(0.76)\end{array}$ \\
\hline $25 \%$ & 191 & 44 & 90 & 96 \\
\hline $50 \%$ & 344 & 75 & 168 & 168 \\
\hline $75 \%$ & 496 & 101 & 240 & 252 \\
\hline $\begin{array}{r}\text { Máximo d } \\
\text { (Camarasa-Belmo }\end{array}$ & $\begin{array}{l}\text { referencia } \\
\text { te y Soriano. 2014) }\end{array}$ & 101 & 337.6 & 413.6 \\
\hline
\end{tabular}

Tabla 2. Umbrales de precipitación máxima acumulada, en diferentes intervalos de tiempo, en relación con los daños. Fuente: elaboración propia.

Si cotejamos los umbrales que producen los mayores daños $(>75 \%)$ con los máximos de referencia, encontramos que mientras en $1 \mathrm{~h}$ el umbral está próximo a este máximo, en 6 h. y 12 h. las cantidades distan bastante. Estos resultados ponen de manifiesto cómo, para intervalos inferiores a una hora, el parámetro más importante es el de intensidad y los daños importantes tienen lugar cuando se alcanzan cantidades similares a los máximos de referencia. Sin embargo, si la lluvia se mantiene durante un período de al menos 6 horas, el efecto acumulativo adquiere relevancia frente al de intensidad, de manera que con cantidades inferiores a los máximos de referencia se pueden producir daños severos. 


\subsection{Comparación entre los umbrales de lluvia obtenidos y los del Plan Meteolaerta}

En general, los umbrales de lluvia en los que se basa el Plan Meteoalerta están considerados sobredimensionados, en el sentido de que están vinculados a un pronóstico de daños superior al que realmente tiene lugar.

La tabla 3 recoge el porcentaje de sobredimensión registrado para cada una de las zonas de estudio. Lo primero que se pone de manifiesto es que, si bien existe un sobredimensionamiento general de las alertas, éste no es igual en los tres niveles. El rojo es el que menor anomalía presenta (exceptuando el área de Gandía para la lluvia de 12 horas), lo que evidencia que los avisos de los episodios de mayor riesgo están bien dimensionados. En general, los desajustes más importantes afectan en mayor medida al nivel amarillo que al naranja y más frecuentemente al intervalo de $1 \mathrm{~h}$ que al de $12 \mathrm{~h}$.

\begin{tabular}{|l|c|c|c|c|c|c|}
\hline \multirow{2}{*}{ ZONA } & \multicolumn{2}{|c|}{ ALERTA AMARILLA } & \multicolumn{2}{c|}{ ALERTA NARANJA } & \multicolumn{2}{c|}{ ALERTA ROJA } \\
\cline { 2 - 7 } & $\mathbf{1 h}$ & $\mathbf{1 2 h}$ & $\mathbf{1 h}$ & $\mathbf{1 2 h}$ & $\mathbf{1 h}$ & $\mathbf{1 2 h}$ \\
\hline Valencia & $57 \%$ & $50 \%$ & $38 \%$ & $14 \%$ & $0 \%$ & $0 \%$ \\
\hline Castellón & $61 \%$ & $56 \%$ & $37 \%$ & $25 \%$ & $0 \%$ & $0 \%$ \\
\hline Benageber & $74 \%$ & $50 \%$ & $71 \%$ & $100 \%$ & - & - \\
\hline Alzira & $62 \%$ & $56 \%$ & $47 \%$ & $17 \%$ & - & $0 \%$ \\
\hline Cofrentes & $65 \%$ & $33 \%$ & $33 \%$ & - & - & - \\
\hline Alicante & $54 \%$ & $50 \%$ & $25 \%$ & $43 \%$ & - & $0 \%$ \\
\hline Gandía & $57 \%$ & $50 \%$ & $33 \%$ & $68 \%$ & - & $20 \%$ \\
\hline
\end{tabular}

Tabla 3. Porcentaje de sobredimensionamiento de las alertas según los umbrales del Plan Meteoalerta. Fuente: elaboración propia

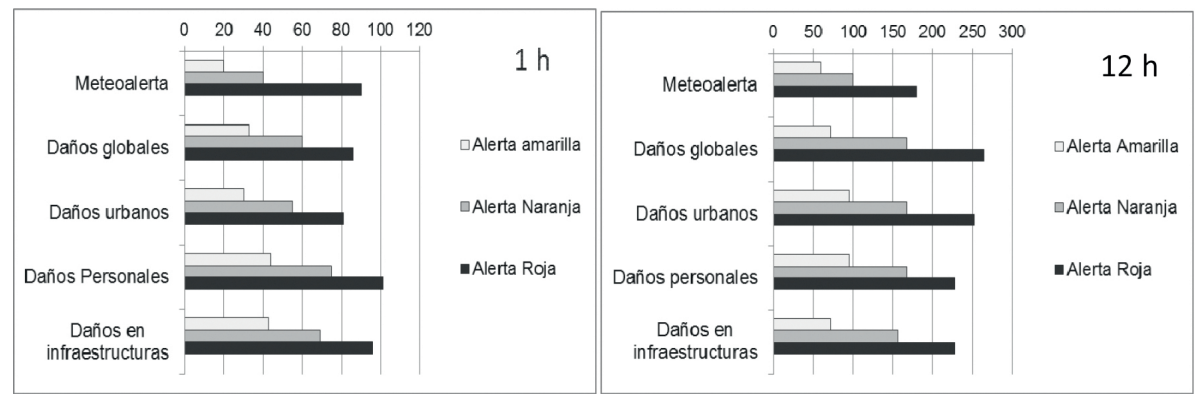

Figura 2. Comparación entre los umbrales de lluvia del Plan Meteoalerta y los que han producido daños en la Comunidad Valenciana entre 1997 y 2007.

La figura 2 compara los umbrales de alerta del Plan Meteoalerta con los que obteníamos en apartado anterior, para niveles de pérdidas del 25\%, 50\% y 75\%, que podríamos hacer equivaler con los colores amarillo, naranja y rojo.

En ella se pone de manifiesto cómo casi todos los casos los daños leves se han producido con umbrales de lluvias mayores a las que propone AEMET (con la única excepción de la alerta roja de $1 \mathrm{~h}$ ). La tabla 4 compara los umbrales de alerta vigentes con los umbrales mínimos de daño estimados en el presente estudio. En ella se pone 
de manifiesto cómo, exceptuando dicho caso de alerta roja para lluvias de 1 hora, todos los demás umbrales podrían ajustarse al alza, sobre todo, para lluvias acumuladas en 12 horas.

\begin{tabular}{|l|c|c|c|c|}
\hline \multirow{2}{*}{ ALERTAS } & \multicolumn{2}{|c|}{$\begin{array}{c}\text { Umbrales de lluvia según } \\
\text { Meteoalerta (mm) }\end{array}$} & \multicolumn{2}{c|}{$\begin{array}{c}\text { Umbrales estimados en este } \\
\text { trabajo (mm) }\end{array}$} \\
\cline { 2 - 5 } & Lluvia 1 h. & Lluvia 12 h. & Llluvia 1 h & Lluvia 12 h \\
\hline Alerta amarilla & 20 & 60 & 30 & 72 \\
\hline Alerta naranja & 40 & 100 & 55 & 156 \\
\hline Alerta roja & 90 & 180 & 81 & 228 \\
\hline
\end{tabular}

Tabla 4. Comparación entre los umbrales de lluvia según el actual Plan Meteoalerta y los estimados en este trabajo. Fuente: elaboración propia

\section{CONCLUSIONES}

La comparación entre indicadores de lluvia y nivel de daños en la Comunidad Valenciana ha presentado buenas correlaciones para la mayor parte de los casos, exceptuando los parámetros de irregularidad y persistencia cincominutal, que han sido desestimados como indicadores de riesgo. Para el resto de parámetros, las mayores pérdidas están correlacionadas en primer lugar con la lluvia total del episodio $\left(\mathrm{r}^{2}=0.92\right)$ $\mathrm{y}$, en menor medida, con la intensidad en una hora $\left(\mathrm{r}^{2}=0.82\right)$ y con las acumuladas en $6 \mathrm{~h}\left(\mathrm{r}^{2}=0.86\right)$ y en $12 \mathrm{~h}\left(\mathrm{r}^{2}=0.84\right)$. Las relaciones más débiles se corresponden con los indicadores cincominutales de intensidad máxima y media.

En términos globales, con lluvias acumuladas de hasta $130 \mathrm{~mm}$ en un episodio se pueden producir daños leves; hasta $298 \mathrm{~mm}$ se esperan daños moderados y, por encima de estas cifras los daños son fuertes. En el análisis por categorías, los umbrales más bajos corresponden a núcleos urbanos, seguido de daños personales y por último de daños en las infraestructuras. Además, mientras para 1 hora los umbrales que producen los mayores daños están próximos a los máximos de referencia, para el caso de lluvias acumuladas en $6 \mathrm{~h}$ y en $12 \mathrm{~h}$, estas cantidades distan bastante.

Estos resultados ponen de manifiesto la importancia de que las lluvias intensas se mantengan durante al menos 6 horas para producir daños. Por tanto, podría ser interesante incluir en los planes de alerta las lluvias acumuladas en 6 horas, como indicador de riesgo (actualmente sólo se considera 1 h y 12 h).

En cuanto al excesivo número de alertas que implican los umbrales vigentes en el Plan Meteoalerta, se ha comprobado que, si bien existe un sobredimensionamiento general, éste es menor en el nivel rojo (los episodios de mayor riesgo están, por tanto, bien recogidos). Los mayores desajustes afectan al nivel amarillo y, en segundo lugar, al naranja y son más frecuentes en el intervalo de $1 \mathrm{~h}$ que en el de $12 \mathrm{~h}$. El estudio comparado de los umbrales que propone el Plan Meteoalerta con los obtenidos en el presente trabajo pone de manifiesto que, con excepción de la alerta roja para 1 hora, todos los umbrales podrían ajustarse al alza, sobre todo, para lluvias acumuladas en 12 horas. 


\section{AGRADECIMIENTOS}

Este trabajo ha sido realizado en el marco del proyecto CGL2013-42728-R, financiado por el Ministerio de Ciencia e Innovación y fondos FEDER). Los autores desean mostrar su agradecimiento al Servicio de Protección Civil de la Delegación del Gobierno en la Comunidad Valenciana (por la cesión de los datos de riesgo); a la Confederación Hidrográfica del Júcar, por la cesión de los datos hidrológicos y al proyecto CGL2007-65368 por la cesión de los indicadores de episodios.

\section{REFERENCIAS}

AEMET, Agencia Española de Meteorología (2009). Meteoalerta. Recuperado de: http://www.aemet.es/es/noticias/2006/07/20060712

Camarasa, A.M. y López-García, M.J. (2006). Criterios de selección y caracterización de episodios de lluvia. Aplicación a la Confederación Hidrográfica del Júcar (19892003). En J.M. Cuadrat, M.A. Sanz, S.M. Vicente, S. Lanjeri, M. de Luis y J.C. González-Hidalgo (Eds); Clima, Sociedad y Medio Ambiente; nº 5, pp. 323-336. Zaragoza, Publicaciones de la AEC, Serie A.

Camarasa-Belmonte, A.M. y Soriano, J. (2014). Empirical study of extreme rainfall intensity in a semi-arid environment at different time scale. Journal of Arid Environments, 100-101, 63-71.

Camarasa, A.M., Soriano, J. y López-García, M.J. (2010). The effect of observation timescales on the characterisation of extreme Mediterranean precipitation, Advances in Geosciences, 26, pp. 61-64. doi: 10.5194/adgeo-26-61-2010

Consorcio de Compensación de Seguros (2014). Estadística. Riesgos Extraordinarios. Serie 1971-2014. Recuperado de: http://www.consorseguros.es/web/ documents/10184/44193/Estadistica_Riesgos_Extraordinarios_1971_2014/14 ca6778-2081-4060-a86d-728d9a17c522

Elizaga, F. (2009). Fenómenos adversos, prioridad básica de AEMET. Meteorología, $85,29-35$

Lorente, M.; Casas, M.C.; Rodríguez, R y Redaño, A. (2013). Intensidades extremas y precipitación máxima probable. En: C. García-Legaz y F. Valero (Eds) Fenómenos Meteorológicos Adversos en España, 142-155. Madrid, AMV Ediciones.

Martín-Vide, J. (1992). Características extremas de la precipitación en la España Mediterránea. Inundaciones y redes de drenaje urbanas, 23-39. Barcelona, Colegio de caminos, canales y puertos.

Martín-Vide, J. (2004). Spatial distribution of daily precipitation concentration index in peninsular Spain, International Journal of Climatology, 24, pp 959-971. doi: $10.1002 /$ joc. 1030

Meteoalarm (2009). Alerting Europe for extreme weather. EUMETNET. Recuperado de: http://www.meteoalarm.eu/es_ES/0/0/ES-Espana.html.

Millán, M.; Estrela, M.J. and Caselles, V. (1995). Torrencial precipitations on the

PATRICOVA: Plan de Acción Territorial de Carácter Sectorial sobre prevención del Rieso de Inundación en la Comunidad Valenciana. Recuperado de: http://www. cma.gva.es/web/indice.aspx?nodo $=76675 \&$ idioma $=$ C.

Saaty, T. (1988). The analytical hierarchy process. New York, McGraw Hill. 